\title{
Обзор методов классификации неподвижных фраз в газовой хроматографии
}

\author{
(C) 2020 Зайцева Е.А. \\ Институт геохимии и аналитической химии им. В.И. Вернадского Российской академии наук \\ (ГЕОХИ РАН), Москва
}

Поступила в редакцию 28.03.2020 г.

DOI: $10.17308 /$ sorpchrom.2020.20/2772

В статье рассматриваются традиционные методы классификации газохроматографических неподвижных фаз. Пятимерная схема Роршнайдера основана на предполагаемой независимости пяти видов энергии у пяти эталонных образцов. Расчет схемы основан на системе уравнений с пятью неизвестными, которые являются характеристиками полярности неподвижной фазы. Однако веществ, у которых был бы только один вид взаимодействия не существует; кроме того, независимых видов межмолекулярной энергии не так много - меньше пяти. Это подразумевает ошибочность и избыточность схемы, поскольку пространство параметров Роршнайдера имеет меньшую размерность, а, принимающиеся независимыми, характеристики эталонных веществ суммируют вклады разных видов энергии. Полуэмпирическая модель сольватационных параметров Абрахама более теоретически обоснована, некоторые ее параметры могут быть получены теоретически. Эта схема еще более сложная в расчетах, чем схема Роршнайдера, а определение всех сольватационных параметров методом газовой хроматографии невозможно. Другие методы используют схемы одномерной характеристики, например, различные параметры полярности или методы гидрофобно-гидрофильного баланса, где неподвижные фазы имеют один оценочный параметр. Этого недостаточно из-за сложной природы межмолекулярных взаимодействий, у неподвижных фаз с различной селективностью характеристики могут совпадать. Традиционные методы эмпиричны, они имеют ограниченную предсказательную способность и не могут одинаково применяться к различным экспериментам. Однако, из-за отсутствия альтернативных вариантов, эти методы широко используются для оценки селективности неподвижных фаз.

Предложен метод трехпараметрической характеристики, который основан на представлении энергии межмолекулярного взаимодействия суммой неполярной, полярной энергии и энергии водородной связи. Все параметры, используемые в математических выражениях, имеют физический смысл, являются переносимыми и не требуют дополнительных экспериментов. Характеристики неподвижных фаз можно рассчитать, как по экспериментальным данным по удерживанию, так a priori, используя структурную формулу неподвижной фазы, если она известна.

Ключевые слова: межмолекулярное взаимодействие, адсорбция, обобщенный заряд, неподвижная фаза, газовая хроматография, полярность.

\section{Введение}

Газовая хроматография (ГХ) позволяет разделять огромное количество различных летучих соединений. В настоящее время для решения аналитических задач разделения методом ГХ, большой популярностью пользуются жидкие неподвижные фазы (НФ). В качестве НФ в колонках наиболее часто используют фазы двух типов: на основе полисилоксанов и на основе полиэтиленгликоля (ПЭГ) [1]. Для каждой хроматографической колонки производитель указывает материал самой колонки, ее длину, внутренний диаметр, толщину жидкой НФ, максимальную температуру экс- 
плуатации, примерный состав и, иногда, некоторые аналогичные фазы. Важной характеристикой НФ является ее полярность. Обратим внимание, что полярность НФ не имеет ничего общего с понятием химической полярности вещества. Полярность вещества - величина дипольного момента его молекул [2], полярность НФ - это способность фазы к удерживанию полярных и поляризуемых соединений [1]. В зависимости от полярности каждая НФ обладает специфической способностью к разделению различных классов химических соединений, то есть обладает различной селективностью по отношению к компонентам разделяемой смеси. Чаще всего в качестве количественной характеристики полярности указывают константы Роршнайдера или МакРейнольдса [3-6] - этот метод является, наверно, самым популярным методом классификации ГХ НФ.

В таблице 1 приведены некоторые коммерческие НФ с их аналогами. Под аналогами понимаются фазы с таким же составом, если он известен, или указанные производителем как аналоги. Такую информацию можно найти на официальном сайте SigmaAldrich, Supelco, Resteck и др. в разеделе «информация о колонке» или «информация для заказа».

Таблица 1. Коммерческие НФ

\begin{tabular}{|c|c|c|c|}
\hline $\begin{array}{c}\text { Название } \\
\text { фазы/колонки }\end{array}$ & $\begin{array}{l}\text { Температура } \\
\text { эксплуатации, } \\
{ }^{\circ} \mathrm{C}\end{array}$ & Состав & Аналоги \\
\hline 1 & 2 & 3 & 4 \\
\hline Stabilwax & $40 \ldots 260$ & ПЭГ & $\begin{array}{c}\text { Supelcowax-10, HP- } \\
\text { Innowax, Carbowax, CP } \\
\text { wax 52 CB, VF-WAX MS, } \\
\text { ZB-WAX Plus, OPTIMA } \\
\text { WAX Plus, AT-WAX, } \\
\text { MXT-WAX }\end{array}$ \\
\hline Stabilwax-DA & $40 \ldots 260$ & ПЭГ & $\begin{array}{l}\text { Nukol, SP-1000, HP- } \\
\text { FFAP, AT-1000, BP-21, } \\
\text { CP WAX 58 CB }\end{array}$ \\
\hline Stabilwax-DB & $40 \ldots 220$ & ПЭГ & $\begin{array}{l}\text { CAM, Carbowax Amine, } \\
\text { CP Wax } 51\end{array}$ \\
\hline Rtx-Wax & $20 \ldots 250$ & $\begin{array}{c}\text { ПЭГ (G14, G15, G16, G20, } \\
\text { G39) }\end{array}$ & $\begin{array}{l}\text { DB-Wax, HP-Wax, AT- } \\
\text { Wax, ZB-WAX }\end{array}$ \\
\hline FAMEWAX & $20 \ldots 250$ & ПЭГ & $\begin{array}{c}\text { Omegawax, AT-AquaWax, } \\
\text { AT-FAME }\end{array}$ \\
\hline OV-1 & 180 & Силиконовое масло & $\begin{array}{l}\text { SE-30, OV-101, Oronite- } \\
\text { OD-1 }\end{array}$ \\
\hline Rxi-1HT & $350 / 400$ & $\begin{array}{c}100 \% \text { полидиметилсилок- } \\
\text { сан (G1, G2, G38) }\end{array}$ & $\begin{array}{c}\text { HP-1, DB-1, VF-1ms, CP } \\
\text { Sil } 5 \text { CB, Ultra 1, DB-1ht, } \\
\text { HP-1ms UI, DB-1ms UI, } \\
\text { ZB-1, ZB-1MS, ZB-1HT } \\
\text { Inferno }\end{array}$ \\
\hline SE-52 & 220 & $\begin{array}{c}\text { (10\% фенил) полиметил- } \\
\text { силоксан }\end{array}$ & OV-3 \\
\hline SE-54 & $350 / 400$ & $\begin{array}{c}\text { (5\% фенил) полиметилси- } \\
\text { локсан }\end{array}$ & $\begin{array}{c}\text { DB-5, SPB-5, BP-5, HP-5, } \\
\text { CPSil-8, Rxi-5HT, Rxi- } \\
\text { 5ms, Rtx-5, HP-5/HP-5ms, } \\
\text { DB-5, ZB-5, ZB-5HT } \\
\text { Inferno, ZB-5ms }\end{array}$ \\
\hline OV-3 & 300 & $\begin{array}{c}\text { (10\% фенил) метилсилок- } \\
\text { сан }\end{array}$ & MS-555, DC-555, DC-556 \\
\hline
\end{tabular}




\begin{tabular}{|c|c|c|c|}
\hline 1 & 2 & 3 & 4 \\
\hline Rxi-5 Sil MS & 350 & $\begin{array}{c}(5 \%(1,4-б и с \\
\text { (диметилсилоксифенилен) } \\
\text { полиметилсилоксан }\end{array}$ & $\begin{array}{c}\text { DB-5ms UI, DB- } 5 \mathrm{~ms} \text {, VF- } \\
5 \mathrm{~ms}, \mathrm{ZB}-5 \mathrm{~ms}\end{array}$ \\
\hline Rtx-35 & 320 & $\begin{array}{c}\text { (35\% дифенил) } \\
\text { полиметилсилоксан (G42) }\end{array}$ & HP-35, DB-35, ZB-35 \\
\hline Rxi-17 & 320 & $\begin{array}{c}\text { (50\% дифенил) } \\
\text { полиметилсилоксан }\end{array}$ & $\begin{array}{l}\text { DB-17ms, VF-17ms, CP } \\
\text { Sil } 24 \mathrm{CB}, \mathrm{ZB}-50\end{array}$ \\
\hline Rxi-624Sil MS & 320 & Запатентованный состав & $\begin{array}{c}\text { DB-624 UI, VF-624ms, } \\
\text { CP-Select } 624 \text { CB }\end{array}$ \\
\hline Rxi-35Sil MS & 360 & Запатентованный состав & $\begin{array}{l}\text { DB-35ms, DB-35ms UI, } \\
\text { VF-35msm, MR2 }\end{array}$ \\
\hline Rxi-XLB & 360 & Запатентованный состав & $\begin{array}{c}\text { DB-XLB, VF-Xms, MR1, } \\
\text { ZB-XLB }\end{array}$ \\
\hline Rtx-1301 & $240 / 280$ & $\begin{array}{c}\text { (6\% цианопропилфенил) } \\
\text { полиметилсилоксан (G43) }\end{array}$ & $\begin{array}{c}\text { Rtx-624, DB-1301, DB- } \\
\text { 624, CP-1301, VF- } \\
\text { 1301ms, VF-624ms, ZB- } \\
624\end{array}$ \\
\hline Rtx-1701 & 280 & $\begin{array}{l}\text { (14\% цианопропилфенил) } \\
\text { полиметилсилоксан (G46) }\end{array}$ & $\begin{array}{l}\text { DB-1701, VF-1701ms, CP } \\
\text { Sil } 19 \text { CB, VF-1701 Pesti- } \\
\text { cides, DB-1701R, ZB- } \\
\text { 1701, ZB-1701P }\end{array}$ \\
\hline Rtx-200 & 340 & $\begin{array}{c}\text { (Трифторпропил) полиме- } \\
\text { тилсилоксан(G6) }\end{array}$ & $\begin{array}{l}\text { Rtx-200MS, DB-200, VF- } \\
\text { 200ms, DB-210 }\end{array}$ \\
\hline Rtx-2330 & 275 & $\begin{array}{c}\text { (90\% бисцианопро- } \\
\text { пил)(10\% цианофенил) } \\
\text { полисилоксан } \\
\text { (G48) }\end{array}$ & VF-23ms \\
\hline Rt-2560 & 250 & $\begin{array}{c}\text { (100\% бисцианопропил) } \\
\text { полисилоксан }\end{array}$ & HP-88, CP Sil 88 \\
\hline Carbowax 300 & $20 \ldots 80$ & ПЭГ м.м. 285-35 & Stabilwax, CP WAX \\
\hline Carbowax 400 & $20 \ldots 100$ & ПЭГ м.м. 380-420 & Stabilwax, CP WAX \\
\hline Carbowax 600 & $30 \ldots 110$ & ПЭГ м.м. 570-630 & Stabilwax, CP WAX \\
\hline Carbowax 1000 & $40 \ldots 120$ & ПЭГ м.м. 950-1050 & Stabilwax, CP WAX \\
\hline Carbowax 1540 & $50 \ldots 140$ & ПЭГ м.м. 1300-1600 & Stabilwax, CP WAX \\
\hline Carbowax 4000 & $60 \ldots 170$ & ПЭГ м.м. 3000-3700 & Stabilwax, CP WAX \\
\hline Carbowax 6000 & $70 \ldots 170$ & ПЭГ м.м. 6000-7500 & Stabilwax, CP WAX \\
\hline $\begin{array}{c}\text { Carbowax } \\
10000\end{array}$ & $70 \ldots 190$ & ПЭГ м.м. 8500-11500 & Stabilwax, CP WAX \\
\hline $\begin{array}{c}\text { Carbowax } \\
15000\end{array}$ & $70 \ldots 220$ & ПЭГ м.м. 13000-17000 & Stabilwax, CP WAX \\
\hline Carbowax 20M & $80 \ldots 250$ & ПЭГ м.м. 15000-20000 & Stabilwax, CP WAX \\
\hline Carbowax 40M & $100 \ldots 250$ & ПЭГ м.м. 35000-40000 & Stabilwax, CP WAX \\
\hline
\end{tabular}

\section{Современная классификация неподвижных фаз}

Характеристика взаимодействия растворенного вещества с растворителем в газожидкостной хроматографии была предметом многочисленных исследований, и сегодня существует множество шкал полярности. В целом они делятся на 2 группы в зависимости от характера измерений удерживания:

- классификация, основанная на абсолютном удерживании и определении термодинамических параметров (здесь рассмотрено не будет). 
- классификация полярности жидкой фазы, основанная на разнице между значениями коэффициента удерживания Ковача [7], измеренными для диапазона исследуемых растворенных веществ, для конкретной НФ и для углеводородной контрольной фазы, обычно сквалана [8].

Индекс удерживания Ковача характеризует удерживание вещества в колонке НФ при температуре Т и рассчитывается относительно двух н-алканов с числом углеродных атомов $m$ и $m+1$ по формуле:

$$
I_{i}=100 \cdot\left(m+\ln \frac{\Gamma_{i}}{\Gamma_{m}} / \ln \frac{\Gamma_{m+1}}{\Gamma_{m}}\right),
$$

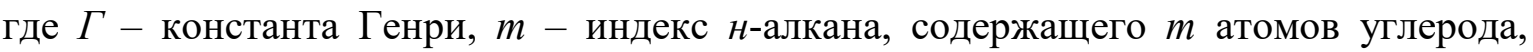
$I_{i}$ - индекс Ковача рассматриваемой молекулы (с индексом $i$ ), для которой выполняется неравенство $\Gamma_{m} \leq \Gamma_{i} \leq \Gamma_{m+1}$.

Индекс Ковача слабо зависит от параметров режима, в частности, от температуры, что позволяет оценивать порядок хроматографического удерживания разных веществ. Численное значение индексов Ковача определяется лишь физико-химическими свойствами анализируемого вещества, природой НФ и температурным режимом колонки, индекс удерживания вещества той или иной НФ, отнесенный к определенной температуре, можно поставить в ряд с такими известными константами, как температура кипения (плавления), плотность или показатель преломления.

Стоит отметить, что большое значение при выборе НФ имеют такие ее свойства, как полярность, способность химически взаимодействовать с исследуемыми веществами, вступать в специфические взаимодействия. Нет необходимости в неограниченном разнообразии НФ, достаточно иметь несколько широко используемых НФ, каждая из которых способна к участию в определенных видах межмолекулярных взаимодействий (ММB), и ряд фаз для решения специальных задач, например, разделение стереоизомеров. При выборе НФ часто руководствуются известным эмпирическим правилом: подобное растворяется в подобном. Качественный учет возможных в системе ММВ часто позволяет осуществлять обоснованный выбор НФ, повысить эффективность разделения и сократить время анализа. Трудность строгого решения этой задачи привела к тому, что в ГХ широко используются различные эмпирические подходы, например, шкалы полярности НФ.

\section{Метод Роршнайдера-МакРейнольдса}

Роршнайдером [3-5] и МакРейнольдсом [6] был предпринят ряд попыток охарактеризовать различные ГХ НФ с помощью эталонных соединений.

Роршнайдер сделал предположение, что характеристикой полярного взаимодействия НФ может служить разность индексов удерживания, найденных на совершенно полярной и неполярной НФ. В качестве эталона неполярной НФ им был выбран сквалан. Для полярного взаимодействия аналита с полярной НФ можно записать выражение:

$$
\Delta I=I_{s p}-I_{s q},
$$

где $\Delta I$ - мера полярности жидкой фазы относительно сквалана, $I_{s p}$ - индекс удерживания эталона на полярной НФ, $I_{s q}$ - индекс удерживания эталона на сквалане.

Далее Роршнайдер предположил, что вклад полярности в индекс удерживания можно описать линейным уравнением вида:

$$
\Delta I=a \cdot x+b \cdot y+c \cdot z+d \cdot u+e \cdot s,
$$

которое содержит ряд констант, зависящих либо от свойств аналитов (константы от $a$ до $e)$, либо от свойств НФ $(x, y, z, u$ и $s)$. Отсутствие алфавитного порядка для пара- 
метров аналита объясняется тем, что изначально Роршнайдер считал достаточным использование только трех параметров - $x, y$ и $z$; позднее он ввел еще два дополнительных параметра.

Роршнайдер выбрал пять эталонных соединений, основываясь на предположении, что каждое из них вступает во взаимодействие с НФ только по одному механизму MМВ и данные виды ММВ независимы друг от друга. В таблице 2 представлены эталонные образцы Роршнадера и их параметры; по схеме Роршнайдера индекс удерживания определяют при $100^{\circ} \mathrm{C}$.

Таблица 2. Эталоны Роршнайдера и их параметры [5].

\begin{tabular}{|c|c|c|c|c|c|c|}
\hline \multirow{2}{*}{$\begin{array}{c}\text { Эталонный } \\
\text { образец }\end{array}$} & $a$ & $b$ & $c$ & $d$ & $e$ & \multirow{2}{*}{$I_{s q}$} \\
\hline бензол & 100 & 0 & 0 & 0 & 0 & 649 \\
\hline этанол & 0 & 100 & 0 & 0 & 0 & 384 \\
\hline метилэтилкетон & 0 & 0 & 100 & 0 & 0 & 531 \\
\hline нитрометан & 0 & 0 & 0 & 100 & 0 & 457 \\
\hline пиридин & 0 & 0 & 0 & 0 & 100 & 695 \\
\hline
\end{tabular}

Параметры подвижной фазы (ПФ) можно найти из индексов удерживания эталонов по следующей формуле:

$$
P=\frac{\Delta I_{E t}}{100},
$$

где $P$ - это соответствующий параметр НФ $-x, y, z, u$ или $s, \Delta I_{E t}-$ мера полярности для эталона на конкретной НФ.

Модификация шкалы Роршнайдера МакРейнольдсом включает использование некоторых более удачных эталонных проб, которые в некоторых вариациях могут быть увеличены до десяти.

Методика определения полярности НФ

Характеристика полярности НФ с помощью метода Роршнайдера-МакРейнольдса включает в себя несколько этапов:

1. Хроматографическое определение индексов Ковача для 5 эталонов на сквалане. Эксперимент проводится в изотермическом режиме $\left(100{ }^{\circ} \mathrm{C}\right)$. В некоторых случаях индексы Ковача эталонов на сквалане могут быть взяты из предыдущих экспериментов или литературы;

2. Хроматографическое определение индексов Ковача для 5 эталонов на исследуемой НФ;

3. Расчет параметров ПФ по формуле (4);

4. Решение системы из 5 уравнений (3) с 5 неизвестными. Полученные константы являются характеристиками полярности НФ.

Система Роршнайдера-МакРейнольдса позволяет [7]: классифицировать ГХ НФ в соответствии с их полярностью и специфическими взаимодействиями, сравнивать различные НФ, выбрать ограниченное число подходящих НФ для широкого применения.

Недостатки метода Роршнайдера-МакРейнольдса

1. Эмпиричность метода. Шкала Роршнайдера не имеет теоретического обоснования, соответственно, имеет ограниченную предсказательную способность и не может быть применена ко всем экспериментам одинаково.

2. Избыточность. Роршнайдер выделяет 5 различных типов взаимодействий у 5 эталонов, приписывая каждому эталону свой тип энергии ММВ и векторно перемножая их, тем самым получая коэффициенты полярности. Получается 5- (а в случае 
использования модифицированной шкалы МакРейнольдса 10-) мерное векторное пространство характеристик, что делает сравнение НФ достаточно сложным.

3. Эталоны. Этот пункт следует напрямую из предыдущего. «Чистых» веществ, у которых был бы только один вид взаимодействия не существует; кроме того, независимых видов межмолекулярной энергии не так много - меньше пяти. Получается, что принимающиеся независимыми характеристики эталонных веществ суммируют вклады разных видов энергии и поэтому не могут служить ортами системы координат.

Метод главных компонент

Сравнение различных НФ довольно сложно с использованием 5 или 10 эталонных аналитов (поскольку оно дает 5- или 10-мерное векторное пространство). Были предприняты многочисленные попытки минимизировать многомерное векторное пространство, сохраняя при этом характерные особенности НФ узнаваемыми. Для этой цели были применены такие методы, как кластерный анализ [9], численная таксономия [10], треугольник Снайдера [11-12] и анализ главных компонентов [13-19]. Анализ основных компонентов [10] также часто применяется для выяснения сходства и различий между поведением удержания растворенных веществ на стационарных фазах в других типах методов хроматографического разделения [18-19].

Результаты работы авторов [13] показывают, что такие особенности, как полярность, поляризуемость и кислотно-основной характер в НФ, могут быть определены с помощью анализа главных компонент. Анализ главных компонент проводился на основе матрицы данных индексов удерживания для 6 аналитов, рассчитанных для 17 НФ. Чтобы избежать возможной потери информации, вызванной нормализацией данных, анализ главных компонент проводился на ковариационной матрице. Расчет был выполнен с $99.99 \%$ объясненной дисперсии.

На рисунке 1 представлен двумерный график переменных, участвующих в анализе главных компонент. Первый главный компонент представлен в виде оси абсцисс, а второй компонент - ординатой с положительными значениями для кислотных фаз и отрицательными значениями для основных фаз. Первый главный компонент практически соответствует полярности НФ. Второй главный компонент больше соответствует кислотно-основным свойствам Льюиса с положительными коэффициентами для кислых НФ. Корреляции между первым и вторым главным компонентом обнаружено не было.

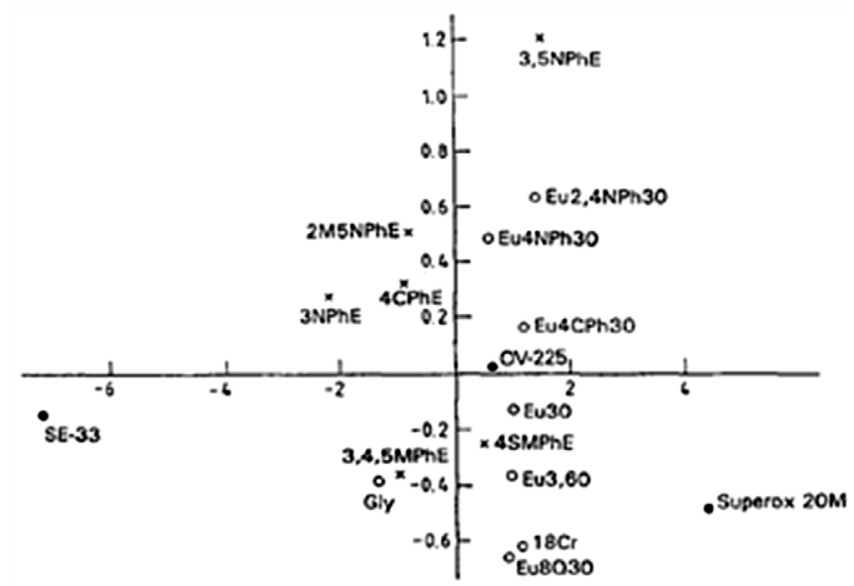

Рис. 1. Двумерная линейная карта НФ. Символы: (•) - контрольные фазы, $(\times)$ - замещенные фенильные фазы, (о) - олигоэтиленоксид и фазы, содержащие эвгенол [13]. 
Классификация ГХ НФ с помощью анализа главных компонент, как видно из рисунка 1, очень наглядна, однако сложность его реализации, необходимость в проведении экспериментов по определению индексов Ковача и зависимость от набора тестовых аналитов делают его крайне неудобным.

\section{Модель сольватационных параметров Абрахама}

Полуэмпирическая модель сольватационных параметров Абрахама внешне схожа со схемой Роршнайдера, однако некоторые ее параметры могут быть рассчитаны теоретически. Символьное представление модели сольватационных параметров, введенное Абрахамом, выглядит следующим образом:

$$
S P=c+e E+s S+a A+b B+v V(l L)
$$

где $S P$ - любая величина, связанная с распределением вещества, например, коэффициент распределения; $E, S, A, B$ и $V$ - так называемые дескрипторы аналита, или независимые переменные, присваиваемые каждому соединению и характеризующие его поляризуемость, дипольность, способность участвовать в образовании водородных связей (Н-связь) в качестве донора и акцептора протона соответственно; молекулярный размер соединения учитывается посредством дескрипторов $V$ при изучении распределения между конденсированными фазами и $L$, если одной из фаз является газ; коэффициенты $e, s, a, b$ и $v, l$ - так называемые сольватационные параметры отражают свойства системы, в которую помещено вещество.

Модель сольватационных параметров была разработана для описания растворения незаряженных соединений в однофазных средах и изучения удерживания, сорбции и экстракции названных типов аналитов в двухфазных системах. Поведение того или иного соединения в подобных системах в основном определяется его склонностью вступать в ММВ с молекулами среды.

Очевидно, что учет всех видов ММВ на практике невозможен, что может приводить к перекрыванию вкладов различных взаимодействий при расчете сольватационных параметров и, как следствие, уменьшению точности описания экспериментальных данных. Однако, Абрахамом [20] и другими исследователями [21] была показана достаточность учета 4 параметров для ММВ и одного параметра для размера молекул аналитов для описания поведения широкого круга веществ в различных по природе двухфазных системах.

Дескрипторы аналита в модели сольватационных параметров не являются эмпирически, произвольно, подобранными величинами, позволяющими точно предсказывать удерживание в определенной системе. Они были введены для исследования различных распределительных систем и соединений, в частности с целью выявления предпочтительного рода взаимодействий, которые реализуются в данной системе, либо для определенного аналита. В таблице 3 приведены дескрипторы аналита с их интерпретацией.

Таблица 3. Дескрипторы аналита модели Абрахама, их обозначение и интерпретация.

\begin{tabular}{|c|c|c|}
\hline Дескриптор & Обозначение & Что описывает \\
\hline 1 & 2 & 3 \\
\hline Поляризуемость & $E$ & $\begin{array}{c}\text { дисперсионные и частично индукционные ван- } \\
\text { дер-ваальсовы взаимодействия }\end{array}$ \\
\hline $\begin{array}{c}\text { Дескрипторы моляр- } \\
\text { ного размера }\end{array}$ & $V$ & $\begin{array}{c}\text { энергия «образования полости» } \\
\text { (эндоэнергетический эффект разрушения связей } \\
\text { растворитель-растворитель) }\end{array}$ \\
\cline { 2 - 3 } & $L$ & $\begin{array}{c}\text { эндоэнергетический эффект + взаимодействия } \\
\text { посредством дисперсионных сил («размерный» } \\
\text { дескриптор) }\end{array}$ \\
\hline
\end{tabular}

Зайцева Е.А. / Сорбционные и хроматографические процессы. 2020. Т. 20. № 2. С. 175-196 


\begin{tabular}{|c|c|c|}
\hline 1 & 2 & 3 \\
\hline Полярность & $S$ & $\begin{array}{c}\text { ориентационные и индукционные } \\
\text { взаимодействия }\end{array}$ \\
\hline Водородные связи* & $A$ & $\begin{array}{c}\text { способность аналита взаимодействовать посред- } \\
\text { ством образования Н-связей в качестве донора }\end{array}$ \\
\cline { 2 - 3 } & $B$ & $\begin{array}{c}\text { способность аналита взаимодействовать } \\
\text { посредством образования Н-связей в качестве } \\
\text { акцептора }\end{array}$ \\
\hline
\end{tabular}

*в работе [22] приводятся эмпирические уравнения для дескрипторов Н-связей, линейно связанных с логарифмическими константами равновесия процесса образования Н-связи $(\log K)$ в тетрахлорметане как для набора соединений-акцепторов Н-связи, так и доноров.

Параметры $e, s, a, b$ и $v$ отражают различие в свойствах двух фаз, между которыми распределяется вещество, либо свойства растворителя, в который оно помещено по тому же типу взаимодействия, что и стоящий рядом дескриптор в уравнении модели Абрахама [21].

\section{Физико-химический смысл дескрипторов и параметров}

Исходя из строения вещества и его коэффициента преломления, возможен прямой расчет дескриптора $E$ для любого необходимого аналита без необходимости проведения эксперимента, либо введения некоторой «приблизительной оценки». Избыточная молярная рефракция, $E$, в уравнении модели сольватационных параметров позволяет учесть взаимодействия растворитель - аналит, основанные на поляризации электронов молекулы аналита, то есть дисперсионные и частично индукционные Вандер-Ваальсовы взаимодействия.

Аналогично $E$, дескриптор молекулярно объема, $V$, рассчитывается напрямую по аддитивной схеме МакГована из атомных фрагментов и числа связей в молекуле. Существует алгоритм для расчета $V$ [22].

В случае распределений, где в качестве одной из фаз является газ, наилучшим образом процесс образования полости учитывается посредством дескриптора $L$, что связано с тем, что в данных системах дисперсионные взаимодействия являются доминирующими [21]. Дескриптор $L$ был определен изначально как логарифм константы равновесия процесса распределения «газ-гексадекан» при $25^{\circ} \mathrm{C}, \log L^{16}$.

Дескриптор полярности, $S$, отражает способность молекул аналита взаимодействовать посредством постоянных, либо наведенных диполей, учитывая, таким образом, ориентационные и индукционные взаимодействия. При рассмотрении дескриптора поляризуемости,можно говорить лишь о его изменении в ряду соединений, но не об его абсолютном значении.

Способность аналита взаимодействовать посредством образования Н-связей в качестве донора и акцептора водорода учитывается дескрипторами $A$ и $B$, соответственно. Разработкой соответствующих шкал занимался Абрахам с коллегами; они исследовали данные МакРейнольдса, который определил объемы удерживания и индексы удерживания (I) для 376 растворенных веществ на 77 НФ при двух температурах [23] и индексы удерживания 10 растворенных веществ на $226 \mathrm{HФ} \mathrm{при} 120^{\circ} \mathrm{C}$ [24].

Сольватационные параметры рассчитываются методом линейной регрессии по результатам экспериментов по распределению, либо удерживанию аналитов в системе; сльватационные параметры обладают физико-химическим смыслом, позволяющим проводить сравнение и анализ природы взаимодействий в интересующих исследователя системах.

Самым неоднозначным и необоснованным слагаемым уравнения Абрахама с физико-химической точки зрения является системная константа $c$. Ее физический 
смысл, с одной стороны, можно представить как гипотетическую модификацию циклогексана, все дескрипторы которого равны нулю. Соответственно, такое соединение не нарушает структуры растворителей, не образует Н-связей и не поляризуется при воздействии внешних диполей [21]. С другой стороны, все взаимодействия реализуемые в конкретном растворителе или системе, точное выделение и учет которых в модели пока не представляется возможным, будут вырождаться именно в системную константу $c$. Потому в анализе уравнения Абрахама, как правило, данная системная константа не участвует. Большой вклад в суммарную величину коэффициента распределения, а также сильная зависимость от набора тестовых соединений, по которому производился расчет сольватационных параметров модели наблюдается для многих распределительных систем, что в значительной степени затрудняет сравнение их свойств между собой.

Численный расчет дескрипторов

Определение большинства дескрипторов зачастую требует проведения предварительных экспериментов, хотя существуют и теоретические подходы к решению данной задачи. Последние, однако, обладают меньшей точностью и так или иначе должны быть соотнесены с экспериментальными значениями. Поэтому в данном разделе будут рассмотрены экспериментальные способы получения дескрипторов, а также методы их оптимизации, в основе которых лежит усреднение уже рассчитанных дескрипторов.

Получение начального массива данных дескрипторов аналитов,а также выявление особенностей их расчета повлекло за собой огромное количество работ по изучению различных систем в рамках модели Абрахама. В настоящее время список охарактеризованных систем достаточно велик и включает в себя ВЭЖХ и ГХ системы, и экстракционные системы «вода-растворитель» и двухфазные системы «растворитель-растворитель» для нерастворимых в воде соединений [25].

Изучение новой системы в рамках модели Абрахама сводится к следующим этапам:

1. Выбор тестовых аналитов - ключевой этап, оказывающий влияние на универсальность и точность будущего уравнения системы.

Главным критерием здесь, конечно, является наличие надежно установленных величин дескрипторов для этих соединений, однако, это обязательное, но недостаточное требование. Необходимо также чтобы величины дескрипторов тестовых аналитов охватывали широкий диапазон значений, т.е. должны существенно различаться по своей химической природе и включать в себя как алифатические, так и ароматические соединения. Авторами [26] было проведено теоретическое исследование списка опубликованных к 2002 году дескрипторов почти 3000 аналитов с помощью метода главных компонент для обоснованного выбора представительного набора аналитов (71 соединение). Кроме того, дескрипторы аналитов, составляющих набор, не должны коррелировать между собой. Это может значительно осложнить определение вкладов, вносимых различными взаимодействиями в результирующую зависимую переменную (например, $\log P$ ), В случае, если в наборе проявляются сильные линейные зависимости между величинами некоторых дескрипторов, необходимо добавить в набор аналиты, чтобы эти корреляции ослабить [22].

2. Проведение эксперимента с целью получения данных об удерживании, либо распределении этих аналитов в системе.

Важным для хроматографических исследований является требование к значениям коэффициента емкости $k$ ': он должен охватывать широкий диапазон значений. Малые значения $k^{\prime}$ крайне чувствительны к ошибкам, связанным с установлением мертвого объема, а это означает, что значения $k^{\prime}$ менее 1 не могут 
рассматриваться как надежные. Рекомендаций относительно конкретного количества соединений в наборе для поиска уравнения модели в литературе нет, но логично предположить, что чем больше данных об удерживании или распределении соединений различных классов будет положено в построение модели, тем более точной она будет.

Как правило, на этом (экспериментальном) этапе набор тестовых аналитов, отобранных по некотороым априорным соображениям, значительно сокращается - по практическим причинам. Например, авторы [27] предварительно отобрали 61 соединение для изучения 3 ВЭЖХ систем «силикагель $\mathrm{C}_{8}$ - вода-ацетонитрил», «силикагель $\mathrm{C}_{8}$ - тетрагидрофуран» и «силикагель $\mathrm{C}_{8}-$ метанол». В итоге для расчета сольватационных параметров для этих систем были взяты 57, 57 и 39 соединений, соответственно; авторы [28] использовали всего 22 тестовых аналита для описания 6 систем.

3. Регрессионная обработка полученных данных.

Финальная обработка массива данных по удерживанию аналитов совместно с пятью дескрипторами $(E, S, A, B$ и $V)$ включает в себя поиск параметров методом многопараметрического анализа, соответствующих статистических показателей (стандартное отклонение для уравнения в целом и параметров в частности, $R^{2}$, коэффициент Фишера), а также проведение тестов на устойчивость модели к изменению числа экспериментальных данных, поиск промахов.

Хроматографические методы, в частности газовая и высокоэффективная жидкостная хроматография, хорошо зарекомендовали себя применительно к этому направлению, ввиду широкого распространения хроматографического оборудования. Кроме того, они требуют малого количества образца, и выдвигают не такие жесткие требования к чистоте, как в методе определения дескрипторов по растворимости.

Сольватационные параметры известны более чем для 60 капиллярных колонок для газовой хроматографии в температурном интервале от 60 до $240{ }^{\circ} \mathrm{C}$ [29]. Такое разнообразие позволяет выбирать наиболее благоприятные условия для определения интересующего дескриптора в зависимости от значения соответствующего параметра в уравнении модели Абрахама. То есть, наиболее точному определению дескрипторов способствует значимо отличное от нуля значение сольватационного параметра. Параметры газохроматографических систем, представленные в работе [30], варьируются в пределах $0.2-0.6,1.3-0,2.9-0$ и $0.8-0.2$ для $e$-, $a$-, $s$ - и $l$-параметров соответственно. Значение параметра $b$ остается нулевым вне зависимости от температуры и природы НФ, а дескриптор $E$ рассчитывается на основании коэффициента преломления. Получается, что метод ГХ наиболее удобен для расчета трех дескрипторов: $A, S$ и $L$.

На основании приведенных рассуждений о соотношении параметров хроматографической системы авторы [31] отмечают НФ, наиболее подходящие для определения одного или нескольких дескрипторов. Так, наилучшей для определения $L$ является колонка с полиметилсилоксаном, так как вклад в удерживание аналита будут вносить только $L$ и $E$. Ввиду большого отношения $s / a$ колонки с поли(метилтрифторопропилсилоксаном) полезны при определении $S$, а с ПЭГ, напротив, для определения $A$ (большое отношение $a / s$ ). Также для совместного определения $A$ и $S$ применяются НФ, содержащие цианоалкилсилоксан, которые являются сильными диполями и поляризуются, а также основаниями приобразовании Н-связей.

Определение дескриптора $B$ посредством ГХ пока невозможно, так как нет колонок со значением сольватационного параметра $b$, значимо отличного от 0 . Однако некоторые попытки синтезировать подходящие НФ для газовой хроматографии, проявляющие кислотность при образовании Н-связей, были вполне успешны. К примеру, 
полидиметилсилоксан, с включениями мономера 2-(бутилфенил)-1,1,1,3,3,3-гексафторопропан-2-ол-метилсилоксана $\left(b=1.75\right.$ при $\left.100^{\circ} \mathrm{C}\right)$ [32] и 4-додецил- $\alpha, \alpha$-бис (трифторметил)бензилового спирта ( $b=2.69$ при $\left.80^{\circ} \mathrm{C}\right)$ [33]. Однако, ни одна из них не является коммерчески доступной. Поэтому, определение дескриптора $B$ методами ГХ пока невозможно.

Таким образом, метод газовой хроматографии позволяет получить наиболее точное относительно других методов значение дескрипторов $A, S$ и $L$. Однако, ГХ имеет недостатки: невозможен расчет дескриптора $B$, выбор аналитов ограничен летучими соединениями.

При определении дескрипторов применяют обратно-фазовую (ОФ) ВЭЖХ. Общим для таких систем является близость к нулю сольватационных параметров $s$ и $a$, а также сильно отличное от нуля значение параметра $b$. Таким образом, с наименьшей неопределенностью методом ОФ ВЭЖХ можно определить дескриптор $B$. Однако при помощи данного метода находят и дескрипторы $S$ и $A$. Но в этом случае стандартное отклонение больше, чем в ГХ.

Нормально-фазовая ВЭЖХ предпочтительна для определения дескриптора $S$, хотя в этих системах, как и в ОФ ВЭЖХ, имеет место ненулевое значение сольватационных параметров $a$ и $b$ [33].

Для повышения достоверности экспериментального определения дескрипторов необходимы данные об удерживании аналитов в нескольких хроматографических системах. Важным принципом здесь является то, что эти системы должны сильно отличаться друг от друга по природе взаимодействий с аналитами, то есть обладать различными по значению сольватационными параметрами.

\section{Недостатки модели Абрахама как способа классификации ГХ НФ}

1. Эмпиричность. Модель сольватационных параметров является полуэмпирической, большая часть параметров определяется из эксперимента. При анализе системы с помощью модели сольватационных параметров необходима экспериментальная проверка теоретически полученных параметров.

2. Избыточность. К избыточности модели Абрахама можно отнести как наложение некоторых вкладов ММВ в дескрипторах аналитов, так и огромное количество тестовых аналитов и предварительных экспериментов.

3. Тестовые аналиты. Выбор набора тестовых аналитов с минимальной корреляцией дескрипторов между собой очень важен для построения устойчивой модели, на основании которой возможно судить о природе сил, за счет которых происходит распределение аналита в системе. В случае, если в наборе проявляются сильные линейные зависимости между величинами некоторых дескрипторов, необходимо добавить в набор аналиты, чтобы эти корреляции ослабить [21]. В этом же пункте следует указать огромное количество тестовых аналитов, используемых для описания 1 системы. Даже с учетом того, что при проведении экспериментальной части работы число тестовых аналитов сокращается, их остается достаточно большое количество - минимум 22 соединения, как было указано выше, для расчета всех дескрипторов и сольватационных параметров. Также стоит отметить, что тестовые аналиты подбираются, исходя из априорных представлений об их природе, по общим требованиям, строго списка тестовых аналитов и их дескрипторов нет, все рассчитывается для конкретного эксперимента. В некоторых случаях можно воспользоваться справочными данными по дескрипторам аналита, которые были ранее рассчитаны Абрахамом и др., но такие данные требуют проверки в конкретной описываемой системе. 
4. Модель сольватационных параметров редко применяется для прогноза времен удерживания при оптимизации хроматографических процессов. Уравнение учитывает не все возможные специфические взаимодействия, которые встречаются в газовой хроматографии, например, невозможность определения дескриптора $B$, a между некоторыми параметрами возможны корреляционные зависимости. Энергии, ответственные за распределение аналита, крайне малы, что делает их точный прогноз при помощи любой универсальной модели, которой является и модель Абрахама, крайне непростой задачей.

\section{Одномерные схемы}

\section{Критерий полярности А}

Полярность НФ характеризуется критерием $A$, который является отношением разностей времени удерживания для соседних н-алканов. Высокая точность оценки $A$ дает чувствительный критерий полярности НФ. Оценку легко выполнить, поскольку она требует только оценки различий во времени удерживания для соседних н-алканов [34].

Поведение удерживания растворенного вещества на конкретной НФ является сложной функцией взаимодействий растворенного вещества и растворителя. Хотя эффективные пропорции конкретного взаимодействия (дисперсионное, ориентационное, кислотно-основное) и его температурной зависимости неизвестны, изменения удерживания не могут быть связаны только с одной переменной, и классификация НФ не является строгой. Углеводороды проявляют только один тип взаимодействия, а именно дисперсионное. Их удержание определяется аддитивностью энтальпии, которая отличается для метильной и метиленовой групп. Следовательно, удерживающие свойства н-алканов не полностью коррелируют с числом атомов углерода. Этот факт подтверждается несколькими объемными свойствами н-алканов, такими как свободная энергия, температура кипения, плотность, показатель преломления и скорректированное время удерживания. Разница во времени удерживания между соседними налканами дает вклад только метиленовой группы.

Отношение соседних разностей времени удерживания определяется как критерий полярности $A[34]$ :

$$
A=\frac{\Delta_{n+1}}{\Delta_{n}}
$$

После замены различий во времени удерживания уравнением $\Delta_{n+1}=\frac{t_{M}}{\beta} \cdot e^{\frac{\Delta G_{n+1}}{R T}}-\frac{t_{M}}{\beta} \cdot e^{\frac{\Delta G_{n}}{R T}}$ и деление на $\left(t_{M} / \beta\right) \exp \left(-\Delta G_{n} / R T\right)$, критерий полярности $A$ можно переписать в виде:

$$
A=\frac{\exp \left(-\Delta G_{n+1}+\Delta G_{n}\right)-1}{1-\exp \left(-\Delta G_{n-1}+\Delta G_{n}\right)}
$$

Уравнение 6 демонстрирует физический смысл константы $A$. Оно показывает относительные изменения между соседними н-алканами относительно центрально расположенного н-алкана с $\mathrm{n}$ атомами углерода. Экспоненциальный член выражает вклад одной метиленовой группы. С увеличением доли дисперсионных взаимодействий пары растворенный растворитель изменение свободной энергии метиленовой группы становится больше и, следовательно, А будет увеличиваться. Таким образом, $A$ является большим для неполярных НФ, и с увеличением полярности НФ $A$ уменьшается. 
Неявная форма критерия полярности $A$ НФ выражает структурные и температурные зависимости. Отсюда следует, что

$$
A=a \cdot \exp \left(b T^{-1}\right)
$$

Константа $a$ демонстрирует статические вклады, связанные с общим числом функциональных групп НФ, а константа $b$ представляет динамические вклады, связанные с молекулярными движениями функциональных групп конкретной НФ. Таким образом, с ростом температуры полярность НФ будет увеличиваться.

Критерий полярности $A$ связан с относительным удерживанием в соответствии с уравнением:

$$
A=r_{n} \cdot \frac{r_{n+1}-1}{r_{n}-1}
$$

Под полярностью НФ понимается количественная мера дисперсионных взаимодействий системы метиленовая группа - НФ. Этот критерий является только функцией колебаний НФ [34]. Авторы [34] утверждают, что результаты и выводы их работы согласуются с результатами предыдущих исследований, в которых взаимодействия н-алканов со НФ рассматриваются как наиболее чувствительный и универсальный критерий классификации НФ.

Критерий $A$ различает различные структуры НФ и их температурную зависимость. Критерий $A$ велик для неполярных фаз и низких температур и уменьшается для НФ с большим количеством селективных функциональных групп и с повышением температуры. Критерий $A$ позволяет немедленно контролировать полярность используемой НФ и становится дополнительным контрольным параметром в экспериментальной работе.

Гидрофобно-гидрофильный баланс

Гидрофобно-гидрофильный баланс (ГГБ) хроматографической системы - важная характеристика ММВ модифицированной поверхности сорбентов с молекулами сорбата и подвижной фазой, правильная оценка которой позволяет управлять хроматографическим процессом [35].

Здесь используется свое понятие полярность: полярный сорбент, неполярный сорбат и т.п. Но корректного универсального (общепринятого) определения полярности нет. Под полярность понимают, в первую очередь, наличие в структуре молекулы неионогенных и ионогенных полярных групп (гидроксильных, амино- и нитрогрупп и др.). При этом имеет значение не только наличие этих групп в молекуле, но и их относительно высокая массовая доля - чем меньше массовая доля углеводородного фрагмента, чем полярней группы и чем больше их в молекуле, тем полярнее сорбат. Количественно полярность молекулы сорбата выражается дипольным моментом; в настоящее время имеется большая база данных экспериментальных измерений дипольного момента, разработан целый ряд программ для квантово-химического расчета дипольного момента.

Для характеристики структурных параметров аналитов и оценки баланса их гидрофильных и гидрофобных свойств Шатц предложил использовать параметр $H-$ критерий гидрофобности, который основан на том, что размер молекул аналита определяется в первую очередь числом атомов углерода, а снижение гидрофобности при введении в его молекулу полярной функциональной группы характеризуется для полифункциональных соединений не столько специфической гидрофильностью группы, сколько самим фактором ее наличия [36].

$$
H=n_{h}-4 \sqrt{n_{f}}
$$


где $n_{h}$ - число элементарных гидрофобных фрагментов молекуле, т.е. сумма атомов углерода и галогенов, а $n_{f}-$ число полярных групп. Было принято допущение, что каждый атом галогена эквивалентен по своему влиянию на гидрофобность вещества как одна метиленовая группа, а полярные группы, расположенные в непосредственном соседстве, рассматриваются как одна полярная группа. Гидрофобность атомов галогенов сильно зависит от характера остальной части молекулы и полярность функциональных групп, в соответствии с теорией химического строения, чувствительна к их взаимному расположению, поэтому критерий $H$ не может детально описывать гидрофобность в узких группах веществ, а является универсальной мерой гидрофобности для сравнительного описания веществ, сильно отличающихся по строению.

Шатцем показано, что величина $H$ линейно коррелирует с параметром $\log P$ логарифмом коэффициента распределения вещества в системе 1-октанол - вода. Наряду с экспериментальным методом определения $\log P$, введенным в научный обиход Ганчем и Лео как мера гидрофобности органических соединений, разработаны методы априорного расчета $\log P$ по кладам атомов, структурных фрагментов и функциональных групп. Корпорация ВioВуtе запатентовала оригинальный алгоритм расчета $\log P$, основанный на современных квантово-химических представлениях и принципах. В этом алгоритме, построенном не на статистических данных, а на квантово-химических моделях, учитывается возможность образования внутримолекулярных Н-связей, индуктивный и мезомерный электронные эффекты и стерический фактор, влияющие на гидрофобность соединения. Для того, чтобы отличать величины $\log P$, полученные по этому алгоритму, их обозначают как $C \log P$.

При наличии специализированного программного обеспечения компьютерные расчеты $\log P$ и $C \log P$ не вызывают затруднений. Привлекательность упрощенного критерия Шатца заключается в том, что в отличие от экспериментального определения или сложных алгоритмов расчета $\log P$ и $C \log P$, произвести оценку $Н$ можно даже на калькуляторе.

Недостатки одномерных схем

1. Эмпиричность. Практически все одномерные схемы не имеют теоретического обоснования, требуют проведения специальных экспериментов.

2. Недостаточность. Ввиду сложной природы ММВ одномерные схемы классификации 1 параметра недостаточно для описания селективности НФ, т.е. эти методы малоинформативны. Это противоположно избыточности методов РоршнайдераМакРенольдса и Абрахама.

\section{Метод трехпараметрической характеристики}

Использование теоретических представлений о ММВ для разработки теоретически обоснованного метода характеристики селективности хроматографических фаз является основной темой исследования в работах авторов [37-39]. Развиваемый подход опирается на фундаментальное описание ММВ и лишен недостатков, присущих традиционным методам: не требуются специальные эталонные адсорбаты, отсутствуют математически некорректные процедуры.

Способ описания ММВ в газохроматографической системе использует выражение для общей энергии ММB [40]:

$$
U=U_{n p}+U_{d p}+U_{H},
$$

где $U_{n p}=U\left(Q_{i}, Q_{s p}, r\right)$ - энергия неполярного взаимодействия - это вандерваальсов потенциал типа потенциала Леннард-Джонса, $U_{d p}=U\left(\mu_{i}^{2}, \mu_{s p}^{2}, r\right)$ - энергия поляр- 
ного взаимодействия, которая выражается как сумма ориентационной связи Рейнганума-Кеезома [41-43] и индукционной связи Дебая-Фалькенхагена [44-45], $U_{H}=U\left(n_{H i}, n_{H s p}, r\right)$ - квантов-омеханическая оценка энергии Н-связи [38]. Ионные связи не рассматриваются ввиду того, что для газохроматографической системы такие взаимодействия не характерны.

В развиваемом авторами методе трехпараметрической характеристики (ТПХ) рассматриваются три ключевые характеристики молекул, связанные с поляризуемостью (которая описывается обобщенными зарядами $Q$ ), полярностью (дипольные моменты, $\mu$ ) и способностью к созданию Н-связей (наличие соответствующих атомов и групп, отображаемое параметром $n_{H}$ ). Две последние характеристики участвуют в так называемых специфических взаимодействиях, которые определяют различия в селективности НФ.

Сразу отметим, что теоретические подходы, положенные в основу метода, позволяют связать результат совместного действия всех межмолекулярных сил со структурой взаимодействующих молекул. Таким образом, становится возможным решение прямой и обратной задач описания селективности ГХ НФ. Характеристики могут быть найдены из структурной формулы самой НФ - это предмет прямой задачи описания селективности ГХ НФ. Также характеристики селективности могут быть найдены из экспериментальных данных по удерживанию определенных веществ на изучаемых фазах, что является предметом обратной задачи моделирования ГХ.

\section{Метод характеристики селективности ГХ НФ}

В работе [46] предлагается метод характеристики НФ, который заключается в теоретическом описании ММВ в системе сорбат-сорбент. В методе используются понятия полярности $\left(v_{0}\right)$ и гидрофильности $\left(w_{0}\right)$, являющиеся отношением характеристик полярных и водородных взаимодействий к характеристике неполярного взаимодействия.

Мерой полярности НФ является отношение квадрата ее дипольного момента к обобщенному заряду (отношение полярных сил к неполярным):

$$
v_{0}=\frac{\mu_{s p}^{2}}{Q_{s p}},
$$

а мерой гидрофильности отношение вероятности образования Н-связи фазой к ее обобщенному заряду:

$$
w_{0}=\frac{\bar{n}_{H}}{Q_{s p}}
$$

Эти величины являются параметрами выражения для энергии адсорбции $U_{m, s p}$ молекулы «m» с обобщенным зарядом $Q_{m}$ и дипольным моментом $\mu_{m}$ на НФ (индекс «sp») со стандартной энергией поверхности $U_{0}$ и дипольным моментом $\mu_{s p}$, которые образуют $n_{H}$ Н-связей [37].

\section{Задачи описания селективности НФ}

Прямая задача

Решение прямой задачи сводится к нахождению характеристик НФ из ее структурной формулы. Для этого необходимо: 
1) Выбрать фрагменты молекулярной структуры НФ, которые отображают химический состав и усреднить их характеристики. Для полимеров рассматривали структуру мономера.

2) Для выбранного фрагмента рассчитать обобщенный заряд по формуле, выведенной в работе [47]:

$$
Q_{s p}=\frac{\alpha e}{3}\left(a_{0} E_{1}\right)^{-3 / 2}
$$

где $E_{1}=e \phi-$ средняя энергия, приходящаяся на 1 электрон химической связи в фрагменте НФ, определяемая по формуле $\phi \approx \bar{\phi}=\left(e N_{b}\right)^{-1} \sum_{i=1}^{N_{b}} E_{i} / z_{i}$ [48], коэффициент $\alpha=\left(\frac{V_{0}}{V_{0}-s}\right)^{3 / 4}=\frac{\left(Q^{4 / 3}+s\right)^{3 / 4}}{Q}, s-$ число экранированных электронов;

3) Определить дипольный момент выбранного фрагмента НФ. Величина дипольного момента может быть взята из справочных данных или рассчитана с помощью компьютерных программ молекулярного моделирования, например, Chem3D (пакет CambridgeOffice);

4) Рассчитать полярность НФ $v_{0}$ по (12);

5) Рассчитать показатель Н-связи $n_{H}$ по формуле $n_{H}=\frac{\sqrt{Q_{d}}+\sqrt{Q_{a}}}{2 \sqrt{Q_{\text {mol }}}}$ [48], где индексы: $Q_{m o l}$ - О3 молекулы или мономера жидкой фазы, $Q_{a}$ и $Q_{d}$ - обобщенные заряды акцепторных и донорных атомов молекулы, соответственно. Донором Н-связи будут являться атомы водорода из функциональных групп (например, водород спиртовой группы -ОН), акцепторами - электроотрицательные атомы, не связанные химически с атомом водорода;

6) Рассчитать гидрофильность НФ $w_{0}$ по (13).

\section{Обратная задача}

Решение обратной задачи заключается в расчете характеристик селективности НФ из результатов хроматографического эксперимента.

Очевидно, что без знания структуры прямое определение полярности и гидрофильности НФ невозможно. Однако эти характеристики можно рассчитать из приведенной энергии сорбции, которая определяется из хроматографического эксперимента. В работе [48] приведены формулы для определения безразмерной энергии сорбции и характеристик НФ из экспериментальных данных по индексам удерживания.

Величиной, характеризующей удерживание на исследуемой НФ, являются индексы Ковача (1). Индексы Ковача нормированы на ряд нормальных алканов, т.е. они не содержат в явном виде информацию о неполярном взаимодействии (т.е. обобщенном заряде). Поэтому описание селективности НФ ограничивается относительными характеристиками - полярностью (12) и гидрофильностью (13).

Приведенная энергия адсорбции позволяет связать характеристики селективности с индексами Ковача; в [48-49] представлена связь между энергией адсорбции и индексом Ковача:

$$
u_{i} \approx Q_{i}^{-1}\left(0.06 I_{i}+2\right)^{3 / 4},
$$

где $Q_{i}-$ ОЗ молекулы аналита, $I_{i}-$ индекс Ковача молекулы аналита на исследуемой НФ. 
В работах авторов [37-40, 46-50] проведен анализ выражения (11) и выведены выражения для расчета характеристик селективности - полярности молекулы аналита $v_{i}=\frac{\mu_{i}^{2}}{Q_{i}}$, полярности адсорбента $v_{0}$ и гидрофильности адсорбента $w_{0}$. Выражения для расчета имеют вид:

$$
\begin{gathered}
v_{0 i}=\frac{\sqrt{u_{i}}-1-c v_{i}}{c\left(1+c_{T} v_{i}\right)} ; v_{0}=\frac{\sum v_{0 i} v_{i}}{\sum v_{i}}, \\
S_{i}=\left(\frac{u-B^{2}}{131.3 B^{1 / 3}}\right)_{i}=7.62 \cdot 10^{-3} \frac{u_{i}-\left[1+c\left(v_{i}+v_{0}+c_{T} v_{i} v_{0}\right)\right]^{2}}{\left[1+c\left(v_{i}+v_{0}+c_{T} v_{i} v_{0}\right)\right]^{1 / 3}} ; w_{0}=\frac{\sum S_{i}}{\sum w_{i}},
\end{gathered}
$$

где $i$ - индекс молекул аналита, $c$ и $c_{T}$ - константы, $u_{i}$ - приведенная энергия адсорбции для молекулы.

Стоит отметить, что расчет характеристик полярности и гидрофильности НФ проводится по молекулам аналитов, принадлежащих к разным классам гидрофильности. Молекулы с $n_{H i}=0$ относятся к классу $M_{0}$ и участвуют только в расчете полярности НФ (например, альдегиды и кетоны, сложные эфиры с большими радикалами [39]), а гидрофильные молекулы с $n_{H i}>0$ относятся к классу $M_{1}$ и участвуют в расчете гидрофильности НФ (ярким примером таких молекул являются спирты и карбоновые кислоты).

Методика расчета позволяет определить характеристики НФ по данным всего двух полярных молекул - по одной из множеств $M_{0}$ и $M_{1}$. Для этого необходимо:

1) Получить экспериментальные данные по удерживанию произвольных аналитов на исследуемой НФ. Рассчитать индексы Ковача этих аналитов на исследуемой НФ. Не обязательно проведение эксперимента, могут быть использованы данные из литературы, информация с сайта производителя хроматографической колонки. Часто производителем указываются индексы Ковача или константы Роршнайдера-МакРейнольса для каждой колонки; пересчет констант МакРейнольдса в характеристики селективности подробно представлен в работе авторов [48];

2) Рассчитать О3 и дипольный момент аналитов по их структурной формуле. Определить полярность аналитов;

3) Рассчитать полярность НФ для множества молекул $M_{0}$, по формулам (6);

4) Рассчитать гидрофильность НФ по данным для молекул множества $M_{l}$, по формулам (7).

\section{Карта селективности НФ}

Найденные характеристики НФ (табл. 4) могут быть представлены в виде двумерной карты селективности. На рисунке 2 представлена карта селективности ПЭГ НФ Carbowax с разными молекулярными массами. В работе [47-49] авторами выводится теоретическая зависимость характеристик селективности ПЭГ от молекулярной массы полимера, которой подчиняются и экспериментально полученные характеристики: с ростом молекулярной массы ПЭГ величины характеристик селективности ПЭГ-НФ уменьшаются. В таблице 4 представлены результаты расчета характеристик селективности ПЭГ-НФ. Карта селективности полиэтиленгликолевых и полисилоксановых НФ представлена на рисунке 2.

При построении карты селективности принципиальной разницы, какой способ расчета использовался для нахождения характеристик, нет, могут быть нанесены как экспериментально найденные характеристики, так и результаты априорного расчета. 
Таблица 4. Характеристики НФ.

\begin{tabular}{|c|c|c|c|c|}
\hline \multirow{2}{*}{ Неподвижная фаза } & \multicolumn{2}{|c|}{ Полярность, Д } & \multicolumn{2}{c|}{ Гидрофильность } \\
\cline { 2 - 5 } & a priori & $\begin{array}{c}\text { из эксп. } \\
\text { данных }\end{array}$ & a priori & $\begin{array}{c}\text { из эксп. } \\
\text { данных }\end{array}$ \\
\hline Carbowax 400 & 0.102 & 0.103 & 0.052 & 0.063 \\
\hline Carbowax 600 & 0.096 & 0.095 & 0.048 & 0.055 \\
\hline PEG 600 & 0.096 & 0.110 & 0.048 & 0.061 \\
\hline Carbowax 1000 & 0.090 & 0.107 & 0.045 & 0.058 \\
\hline Carbowax 4000 & 0.083 & 0.078 & 0.038 & 0.044 \\
\hline PEG 4000 & 0.083 & 0.093 & 0.038 & 0.051 \\
\hline Carbowax 6000 & 0.082 & 0.091 & 0.037 & 0.050 \\
\hline Carbowax 20M & 0.081 & 0.090 & 0.034 & 0.049 \\
\hline
\end{tabular}

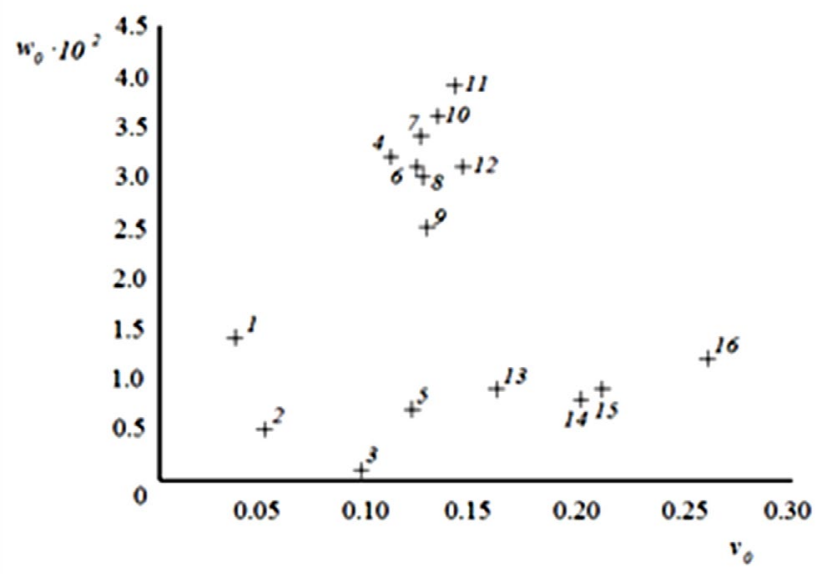

Рис. 2. Карта селективности хроматографических фаз [46] - в координатах полярность $v_{0}\left(\mathrm{D}^{2}\right)$ - гидрофильность $w_{0} \cdot 100$. Фазы представлены в порядке увеличения полярности $v_{0}: 1$ - Сквалан, 2 - SE-30, 3 - OV-7, 4 - Carbowax 6000, 5 - DC-710, 6 - Carbowax 4000, 7 - Carbowax 1540, 8 - Carbowax 20M, 9 - Carbowax 1000, 10 - Carbowax 600, 11 - Carbowax 400, 12 - Carbowax 300, 13 - OV-25, 14 - XE-60, 15 - OV-225, 16 - Silar-5CP.

Карта селективности позволяет выбрать наиболее подходящую для разделения заданной группы аналитов фазу, воспользовавшись эмпирическим принципом «подобное в подобном». Для того, чтобы выбрать подходящую НФ, необходимо рассчитать характеристики полярности и гидрофильности как для НФ, так и для молекул аналитов; характеристики молекул рассчитываются из их структурной формулы, способ расчета характеристик НФ не имеет значения. На карту селективности необходимо нанести характеристики НФ и молекул аналитов, а дальше просто выбрать НФ, наиболее близко расположенную к группе целевых аналитов на карте селективности.

\section{Преимущества метода ТПХ}

1. Фундаментальность. Предложенный метод характеристики ГХ НФ основан на теории $\mathrm{MMB}$, учитывающей неполярные, полярные силы и Н-связи. Для описания неполярных взаимодействий и введения теоретических характеристик применена теория обобщенных зарядов, полярные связи учитываются теориями Рейнганума-Кеезома и Дебая-Фалькенхагена, на основании квантово-механической оценки учитывается вклад в энергию ММВ Н-связей. Все члены уравнения для полной энергии ММВ можно вывести теоретически, отсутствуют эмпирические параметры. Все три вида 
энергии являются взаимонезависимыми, т.е. не происходит наложения и перемножения одинаковых вкладов энергии.

2. Математический расчет. Характеристики селективности НФ достаточно легко получить как из экспериментальных данных, так и рассчитать. a priori, сам pacчет достаточно прост.

3. Отсутствие эталонных аналитов. В методе ТПХ отсутствуют табулированные эталоны, как в методе Роршнайдера, и в то же время нет такой неопределенности в выборе тестовых аналитов как в модели Абрахама. Для получения характеристик селективности из данных хроматографического эксперимента достаточно всего 2 аналитов, принадлежащих разным классам по гидрофильности (1 аналит, относящийся к классу $M_{0}$, и 1 аналит, относящийся к классу $\left.M_{l}\right)$.

4. Наглядность метода. Метод ТПХ позволяет классифицировать НФ в удобном графическом виде - в виде карты селективности.

\section{Список литературы}

1. Stationary Phases in Gas Chromatography. / Rotzsche H. Amsterdam. The Netherlands: Elsevier Science. 1991.409 p.

2. Химия. Справочное руководство. Ленинград: Химия. 1975. $574 \mathrm{c}$.

3. Rohrschneider L. // J.Chomatogr. A. 1965. Vol. 17. pp. 1-12.

4. Rohrschneider L. // J. Chromatogr. 1966. Vol. 22. P. 6-22.

5. Rohrschneider L. // J. Chromatogr. 1969. Vol. 39. P. 383-397.

6. McReynolds W.O. // J.Chromatogr. A. 1970. Vol. 8. pp. 337-345.

7. Схунмакерс П. Оптимизация селективности в хроматографии. М. Мир. 1989. 399 с.

8. Evans M.B., Osborn M.J. // Chromatographia. 1980. Vol. 13. No 3. pp 177-182.

9. DeBeer J.O., Hendrix A.M. // J. Chromatogr. 1982. Vol. 235. pp. 337-349.

10.Huber J.F.K., Reich G. // J. Chromatogr. 1984. Vol. 294. pp. 15-29.

11. Snyder L.R. // J. Chromatogr. 1974. Vol. 92. pp. 223-230.

12.Klee M.S., Kaiser M.A., Laughlin K.B. // J. Chromatogr. 1983. Vol. 279. P. 681-688.

13.Juvancz Z., Cserhaiti T., Markides K.E., Bradshaw J.S. // Chromatographia,. 1994. Vol. 38.pp. 227-231

14. Wolf S., Andersson K. // J. Chromatogr. 1973. Vol. 80. pp. 43-59.

15.McCloskey D.M., Hawkes S.Z. /// J. Chromatogr. Sci. I975. Vol. 13, pp. 1-5.

16.Stark T.J., Larson P.A., Dandeneau R.D. // J. Chromatogr. 1983. Vol. 279. pp. 31-40.

17.Marida K.V., Kent Z.T., Bibby Z.M. Multivariate Analysis, Academic Press, London and New York, 1979.518 p.
18.Cserhati T., Osapay G., Szogyi M. // J. Chromatogr. Sci. 1989. Vol. 2. pp. 540-544.

19.Scerhati T., Valko K. // J. Biochem. Biophys. Methods. 1990. Vol. 20. pp. 81-95.

20.Abraham M.H., Ibrahim A., Zissimos A.M. // J. Chromatogr. A. 2004. Vol. 1037. pp. 29-47.

21.Vitha M., Carr P.W. // J. Chromatogr. A. 2006. Vol. 1126. pp. 143-194.

22.Abraham M.H. // Chem. Soc. Rev. 1993. Vol. 22. pp. 73-83.

23.McReynolds W.O.. Gas Chromatographic Retention Data. Preston. Technical Abstracts Co. Niles. 1966. 335 p.

24.McReynolds W.O. // J. Chromatogr. Sci. 1970. Vol. 8. pp. 685-691.

25.Leahy D.E., Morris J.J., Taylor P.J., Wait A.R. // J. Chem. Soc., Perkin Trans. 1992. Vol. 2. pp. 705-722.

26.Fuguet E., Rafols C., Bosch E., Abraham M.H. et al. // J. Chromatogr. A. 2002. Vol. 942. pp. 237-248.

27.Wang A., Tan L.C., Carr P.W. // J. Chromatogr. A. 1999. Vol. 898. pp. 21-37.

28.Zhao J., Carr P.W. // Anal. Chem. 1998. Vol. 70. pp. 3619-3628.

29.Poole C.F., Poole S.K. // J. Chromatogr. A. 2002. Vol. 965. pp. 263-299.

30.Abraham M.H., Du C.M., Platts J.A. // J. Org. Chem. 2000. Vol. 65. pp. 7114-7118.

31.Lombardo F., Shalaeva M.Y., Tupper K.A., Gao F. et al. // J. Med. Chem. 2000. Vol. 43. pp. 2922-2928.

32.Dias N.C., Poole C.F. // J. Planar. Chromatogr. 2000. Vol. 13. pp. 337-347.

33. Sandi A., Nagy M., Szepesy L. // J. Chromatogr. A. 2000. Vol. 893, pp. 215-234. 
34.Tan L., Carr P.W. // J. Chromatogr. A. 1998. Vol. 799. pp. 1-19.

35.Ševčik J., Löwentap M.S.H. // J. Chromatogr. A. 1981. Vol. 217. pp. 139-150.

36.Долгоносов А.М., Рудаков О.Б., Суровцев И.С., Прудковский А.Г. Колоночная аналитическая хроматография как объект математического моделирования. ГЕОХИ РАН. Воронежский ГАСУ. Воронеж. 2013. 400 с.

37.Сычев С.Н. // Сорбиионные и хроматографические прочессы. 2003. Т. 3. № 4. С. 446455.

38. Долгоносов А.М., Зайцева Е.А. // Сорбиионные и хроматографические прочессы. 2014. T. 14. № 4. С. 578-590.

39.Долгоносов А.М., Зайцева Е.А. // Сорбиионные и хроматографические прочессы. 2015. T. 15. № 3. C. 321-332.

40.Долгоносов А.М., Зайцева Е.А. // Вестник Университета Дубна. 2015. № 1. С.36-41.

41.Долгоносов А.М. // Сорбционные и хроматографические проиессы. 2015. Т. 15. № 3. C. 312-320.
42.Каплан И.Г. Межмолекулярные взаимодействия. Физическая интерпретация, компьютерные расчеты и модельные потенциалы. М. БИНОМ. Лаборатория знаний. 2012. 394 с.

43.Reinganum M. // Ann.d.Physik. 1912. Vol. 38. pp. 649-668.

44.Keesom W.H. // Phys.Z. 1921. Vol. 22. pp. 129-141.

45.Debye P. // Phys.Z. 1920. Vol. 21. pp. 178187.

46.Falkenhagen M. // Phys.Z. 1922. Vol. 23. pp. 87-95.

47.Зайцева Е.А., Долгоносов А.М. // Сорбиионные и хроматографические прочессы. 2018. T. 18. № 5. C. 676-689.

48.Dolgonosov A.M. // Rus. Chem. Bul. 2016. Vol. 65. pp. 952-963.

49.Зайцева Е.А., Долгоносов А.М. // Сорбиионные и хроматографические проиессы. 2019. T. 19. № 5 C. 525-541.

\title{
Review of the methods of stationary phase classification in gas chromatography
}

\author{
(C) 2020 Zaitseva E.A.
}

Vernadsky Institute of Geochemistry and Analytical Chemistry of Russian Academy of Sciences, Moscow

The article discusses the traditional methods of classification of gas chromatographic stationary phases. Rorschneider's five-dimensional scheme is based on the alleged independence of five types of energy in five reference samples. The calculation of the scheme is based on a system of equations with five unknowns, which are characteristics of stationary phase polarity. However, there are no substances with only one type of intermolecular interaction. In addition, there are not so many independent types of intermolecular energy (less than five). This implies the fallacy and redundancy of the scheme, since the Rorschneider parameter space has a smaller dimension, and the independent characteristics of the reference substances summarise the contributions of different types of energy. The semi-empirical Abraham's model of linear solvation energy relationships is more theoretically substantiated. Some of its parameters can be derived theoretically. However, it is even more complicated with regard to calculations than Rorschneider scheme, and the determination of all parameters by gas chromatography is impossible. Other methods use one-dimensional characterisation schemes, for example, various polarity parameters or hydrophobic-hydrophilic balance methods, where the stationary phases have one estimated parameter. This is not enough due to the complex nature of intermolecular interactions. In this regard, the parameters of the characteristic can coincide in stationary phases with different selectivity. Traditional methods have limited predictive power and cannot be equally applied to different experiments. However, due to the lack of alternatives, these empirical methods are widely used to assess stationary phase selectivity.

The article proposes a three-parameter characterisation method, which is based on the representation of the energy of intermolecular interaction by the contributions of non-polar, polar forces, and hydrogen bonds. All parameters used in mathematical expressions have physical meaning, are portable, and do not require additional experiments. The characteristics of the stationary phases can be calculated both from experimental data on chromatographic retention, and a priori, using the structural formula of the stationary phase, if known.

Keywords: molecular interaction, adsorption, generalised charge, stationary phase, gas chromatography, polarity 


\section{References}

1. Stationary Phases in Gas Chromatography, Rotzsche H. Amsterdam, The Netherlands, Elsevier Science, 1991. pp. 409.

2. Khimia, Spravochnoe rukovodstvo, Leningrad, Khimia, 1975, 574 p.

3. Rohrschneider L., J.Chomatogr. A, 1965, Vol. 17, pp. 1-12.

4. Rohrschneider L., J. Chromatogr., 1966, Vol. 22, pp. 6-22.

5. Rohrschneider L., J. Chromatogr., 1969, Vol. 39, pp. 383-397.

6. McReynolds W.O., J.Chromatogr. A, 1970,

Vol. 8, pp. 337-345.

7. Skhunmakers P., Optimizaciya selektivnosti v khromatografii, M., Mir, 1989, 399 p.

8. Evans M.B., Osborn M.J., Chromatographia, 1980, Vol. 13, No 3, pp 177-182.

9. DeBeer J.O., Hendrix A.M., J. Chromatogr., 1982, Vol. 235, pp. 337-349.

10.Huber J.F.K., Reich G., J. Chromatogr., 1984, Vol. 294, pp. 15-29.

11. Snyder L.R., J. Chromatogr., 1974. Vol. 92, pp. 223-230.

12.Klee M.S., Kaiser M.A., Laughlin K.B., $J$. Chromatogr., 1983, Vol. 279, pp. 681-688.

13.Juvancz Z., Cserhaiti T., Markides K.E., Bradshaw J.S., Chromatographia., 1994, Vol. 38 , pp. 227-231.

14.Wolf S., Andersson K., J. Chromatogr., 1973, Vol. 80, pp. 43-59.

15.McCloskey D.M., Hawkes S.Z., J. Chromatogr. Sci., I975, Vol. 13, pp. 1-5.

16.Stark T.J., Larson P.A., Dandeneau R.D., $J$. Chromatogr., 1983, Vol. 279, pp. 31-40.

17.Marida K.V., Kent Z.T., Bibby Z.M., Multivariate Analysis, Academic Press, London and New York, 1979, 518 p.

18.Cserhati T., Osapay G., Szogyi M., J. Chromatogr. Sci., 1989, Vol. 2, pp. 540-544.

19. Scerhati T., Valko K., J. Biochem. Biophys. Methods., 1990, Vol. 20, pp. 81-95.

20. Abraham M.H., Ibrahim A., Zissimos A.M., J. Chromatogr. A, 2004, Vol. 1037, pp. 29-47.

21.Vitha M., Carr P.W., J. Chromatogr. A, 2006, Vol. 1126, pp. 143-194.

22.Abraham M.H., Chem. Soc. Rev. 1993. Vol. 22, pp. 73-83.

23.McReynolds W.O., Gas Chromatographic Retention Data. Preston. Technical Abstracts Co. Niles, 1966, 335 p.

24.McReynolds W.O., J. Chromatogr. Sci., 1970, Vol. 8, pp. 685-691.
25.Leahy D.E., Morris J.J., Taylor P.J., Wait A.R., J. Chem. Soc., Perkin Trans., 1992, Vol. 2, pp. 705-722.

26.Fuguet E., Rafols C., Bosch E., Abraham M.H. et al., J. Chromatogr. A, 2002, Vol. 942, pp. 237-248.

27.Wang A., Tan L.C., Carr P.W., J. Chromatogr. A, 1999, Vol. 898, pp. 21-37.

28.Zhao J., Carr P.W., Anal. Chem., 1998, Vol. 70, pp. 3619-3628.

29.Poole C.F., Poole S.K., J. Chromatogr. A, 2002, Vol. 965, pp. 263-299.

30.Abraham M.H., Du C.M., Platts J.A., J. Org. Chem., 2000, Vol. 65, pp. 7114-7118.

31.Lombardo F., Shalaeva M.Y., Tupper K.A., Gao F. et al., J. Med. Chem., 2000, Vol. 43, pp. 2922-2928.

32.Dias N.C., Poole C.F., J. Planar. Chromatogr., 2000, Vol. 13, pp. 337-347.

33. Sandi A., Nagy M., Szepesy L., J. Chromatogr. A, 2000, Vol. 893, pp. 215-234.

34.Tan L., Carr P.W., J. Chromatogr. A, 1998, Vol. 799, pp. 1-19.

35.Ševčik J, Löwentap M.S.H., J. Chromatogr. A, 1981, Vol. 217, pp. 139-150.

36.Dolgonosov A.M., Rudakov O.B., Surovtsev I.S., Prudkovskii A.G., Kolonochnaya analiticheskaya khromatografiya kak ob'ekt matematicheskogo modelirovaniya. GEOKHI RAN, Voronezhskii GASU, Voronezh, 2013, $400 \mathrm{p}$.

37. Sychev S.N., Sorbtsionnye $i$ khromatograficheskie protsessy, 2003, Vol. 3, No 4, pp. 446-455.

38.Dolgonosov A.M., Zaitseva E.A., Sorbtsionnye i khromatograficheskie protsessy, 2014, Vol. 14, No 4, pp. 578-590.

39.Dolgonosov A.M., Zaitseva E.A., Sorbtsionnye i khromatograficheskie protsessy, 2015, Vol. 15, No 3, pp. 321-332.

40.Dolgonosov A.M., Zaitseva E.A., Vestnik Universiteta Dubna, 2015, No 1, pp. 36-41.

41.Dolgonosov A.M., Sorbtsionnye i khromatograficheskie protsessy, 2015, Vol. 15, No 3, pp. 312-320.

42.Kaplan I.G., Mezhmolekulyarnye vzaimodejstviya. Fizicheskaya interpretaciya, komp'yuternye raschety i model'nye potencialy, BINOM, Laboratoriya znanij, 2012, 394 p.

43.Reinganum M., Ann.d.Physik, 1912, Vol.38, pp. 649-668.

44.Keesom W.H., Phys.Z, 1921, Vol. 22, pp.129-141. 
45.Debye P., Phys.Z., 1920, Vol.21, pp.178187.

46.Falkenhagen M., Phys.Z., 1922, Vol.23, pp.87-95.

47.Zaitceva E.A., Dolgonosov A.M., Sorbtsionnye i khromatograficheskie protsessy, 2018, Vol. 18, No 5, pp. 676-689.

Зайцева Елена Александровна - аспирантка четвертого года обучения, лаборатория сорбционных методов, Институт геохимии и аналитической химии им. В.И. Вернадского РАН (ГЕОХИ РАН), г. Москва.
48.Dolgonosov A.M., Rus. Chem. Bul., 2016, Vol. 65, pp. 952-963.

49.Zaitceva E.A., Dolgonosov A.M., Sorbtsionnye i khromatograficheskie protsessy, 2019, Vol. 19, No 6. pp. 525-541.

Zaitceva Elena A. - PhD student, Lab of Sorption Methods, Vernadsky Institute of Geochemistry and Analytical Chemistry Russian Academy of Sciences (GEOKHI RAS), Moscow. lil-dante@mail.ru 